\title{
VLBA Observations of Blazars
}

\section{Svetlana Jorstad}

Institute for Astrophysical Research, Boston University

725 Commonwealth Ave., Boston, MA 02215, USA

E-mail: jorstadebu.edu

VLBI observations of blazars provide the closest look at compact jets that are thought to be responsible for the variable emission of blazars from radio to $\gamma$-ray wavelengths. It appears that flux variability at different wavelengths is connected to changes in jet structure near its base. The changes happen on timescales of weeks or less, which require frequent VLBI monitoring to follow. Currently, the Very Long Baseline Array is the best tool to perform such observations. Images from the VLBA reveal connections between events in compact jets and behaviour at different wavelengths. Here, I discuss how physical parameters (Lorentz and Doppler factors, viewing and opening angles) of compact jets of blazars can be derived from intense VLBA monitoring at $7 \mathrm{~mm}$, variability of polarization in the core region of parsec scale radio jets, and connections between 7 $\mathrm{mm}$ polarization in the VLBI core and polarization at shorter wavelengths.

Workshop on Blazar Variability across the Electromagnetic Spectrum

Palaiseau, France

April $22^{\text {nd }}-25^{\text {th }} 2008$ 


\section{Introduction}

Blazars form a small group of active galactic nuclei (AGN) that exhibit pronounced variability on both long and short timescales at all wavelengths from radio to $\gamma$-ray frequencies. They feature one-sided jets with knots that move at superluminal apparent velocities. There are strong indications that blazars detected by EGRET possess the most relativistic jets, some with $\beta_{\text {app }}>30 \mathrm{c}$ [1-3]. Explaining the formation of such highly relativistic jets has presented a longstanding challenge for theoretical models and numerical simulations. The current leading explanation for jet production suggests that acceleration and collimation of AGN jets involves poloidal magnetic fields anchored in plasma orbiting a central black hole (e.g., [4], [5]) that dominate the jet energy on scales $\approx(10-1000) \mathrm{R}_{\mathrm{g}}$ (where $\mathrm{R}_{\mathrm{g}}$ is half the Schwarzschild radius). Farther down this region there should be a transition zone where the magnetically dominated jet transforms into a kinetic jet. This transformation zone is most likely located in the zones where far-infrared or submillimeter emission is produced [6]. Currently, the most direct way to deliver observational evidence for such a model is to study the linear polarization of blazars at different frequencies (from radio to optical), along with changes in the innermost jet structure, that will provide the information about the magnetic field structure in different radiation zones.

The unique instrument for studying polarization in the jet on parsec and sub-parsec scales through direct imaging is the Very Long Baseline Array (VLBA), which allows one to monitor dozens of sources with ultra-high angular resolution. In one such study, I and collaborators used the VLBA to obtained total and polarized intensity images of 15 AGNs at $7 \mathrm{~mm}(43 \mathrm{GHz})$ at 17 epochs over three years. The VLBA observations were accompanied at many epochs by nearly simultaneous (within two weeks) measurements of polarization at sub-millimeter and optical wavelengths. In the second half of the program, polarization observations at $3 \mathrm{~mm}$ were performed at several epochs as well. The main results of the project have been pubished in $[7,8]$. Here, I will present the primary findings obtained from this project.

\section{Sample and Observations}

The sample that we observed consists of 8 quasars, 5 BL Lac objects, and 2 radio galaxies (3C 120 of Fanaroff-Riley type 1 and 3C 111 of type 2). Tables 1 and 2 present the list of sources and details about the wavelengths, telescopes, and observational periods of the project.

Table 1. The Sample

\begin{tabular}{lll}
\multicolumn{1}{c}{ Quasars } & BL Lac Objects & Radio galaxies \\
PKS 0420-014 & 3C 66A & 3C 111 \\
PKS 0528+134 & OJ 287 & 3C 120 \\
3C 273 & $1803+784$ & \\
3C 279 & $1823+568$ & \\
PKS 1510-089 & BL Lac & \\
3C 345 & & \\
CTA 102 & &
\end{tabular}


Table 2. The Instruments and Wavelengths

\begin{tabular}{lrl}
\hline VLBA $(7 \mathrm{~mm})$ & March 1998 - April 2001 & 17 epochs \\
BIMA $(3 \mathrm{~mm})$ & April 2000 - April 2001 & $3-4$ epochs \\
JCMT $(0.85 / 1.3 \mathrm{~mm})$ & March 1998 - April 2001 & 6-11 epochs \\
1.5m Steward Obs. $(\sim 6500 \AA)$ & Feb. 1999 - April 2001 & $4-5$ epochs
\end{tabular}

\section{Total and Polarized Intensity Imaging with the VLBA}

The high sensitivity and resolution of the VLBA allowed us to obtain images of all 15 sources with dynamic range $\sim 500: 1$ and resolution $\approx 0.15$ mas. The most prominent features on VLBI images of jets in blazars are the core, which is the bright, very compact section at the narrow end of a one-sided jet (Fig. 1, feature A0), and condensations in the flow that appear as bright knots, often called "components," of the jet (Fig. 1; e.g., components B1 and B2).

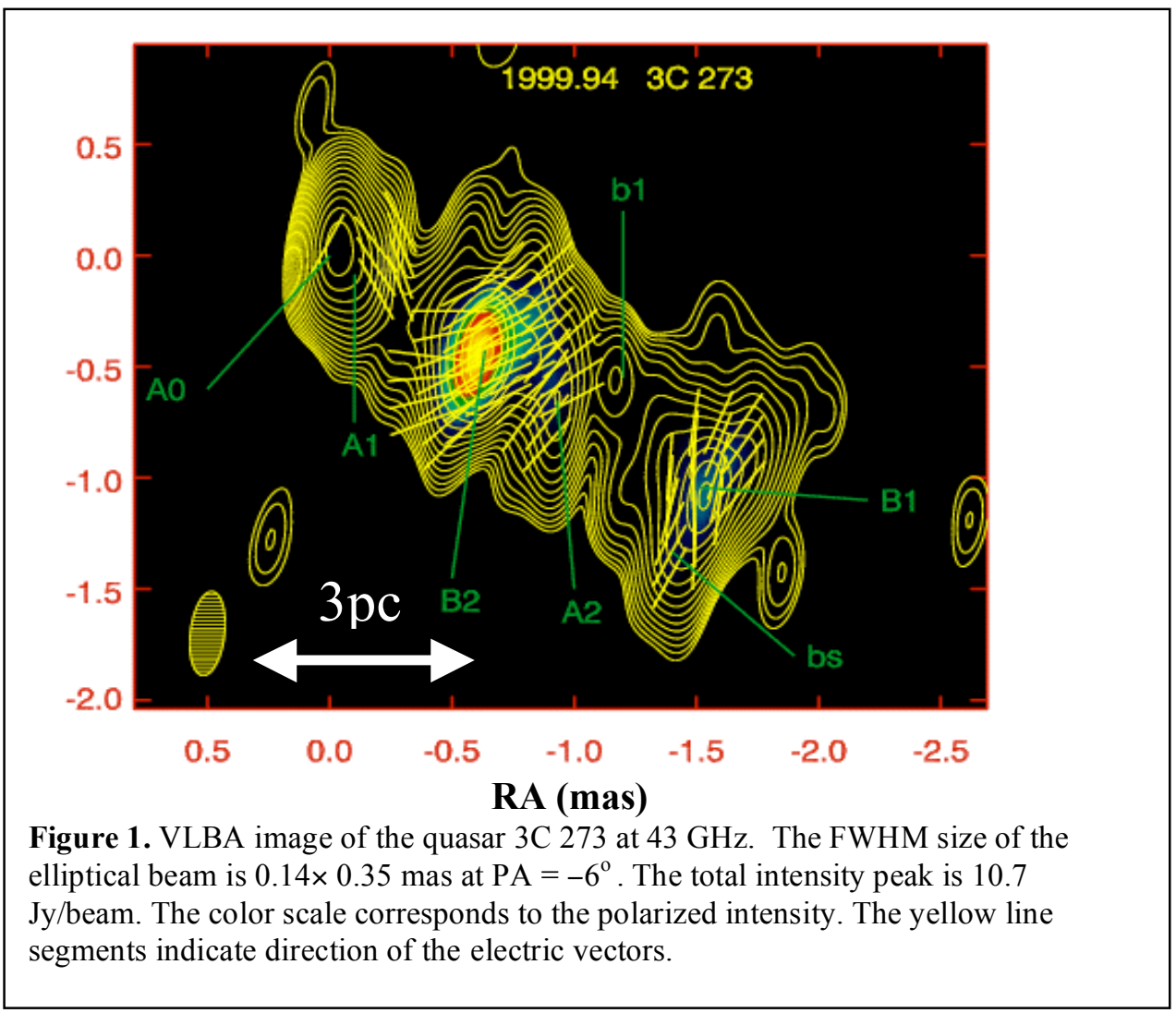

We assume that the core is a stationary feature of the jet and analyze changes in the jet structure relative to the core across epochs. Some components showed obvious motion downstream of the jet (e.g., Fig. 2 left, the bright polarized component in the quasar 3C 273), others did not reveal motion during our observations although changes in brightness or polarization occurred (e.g., Fig. 2 right, the polarized feature about 1 mas from the core in OJ287). To study such behavior in detail, we perform model fitting of components in total and 
polarized intensity images with circular Gaussians. This provides for each component a set of parameters: total intensity flux, $S_{7 \mathrm{~mm}}$, distance from the core, $R$, position angle in the jet in projection on the sky plane, $\Theta$, size of component, $a$, degree of polarization, $m_{7 \mathrm{~mm}}$, and position angle of polarization, $\chi_{7 \mathrm{~mm}}$.
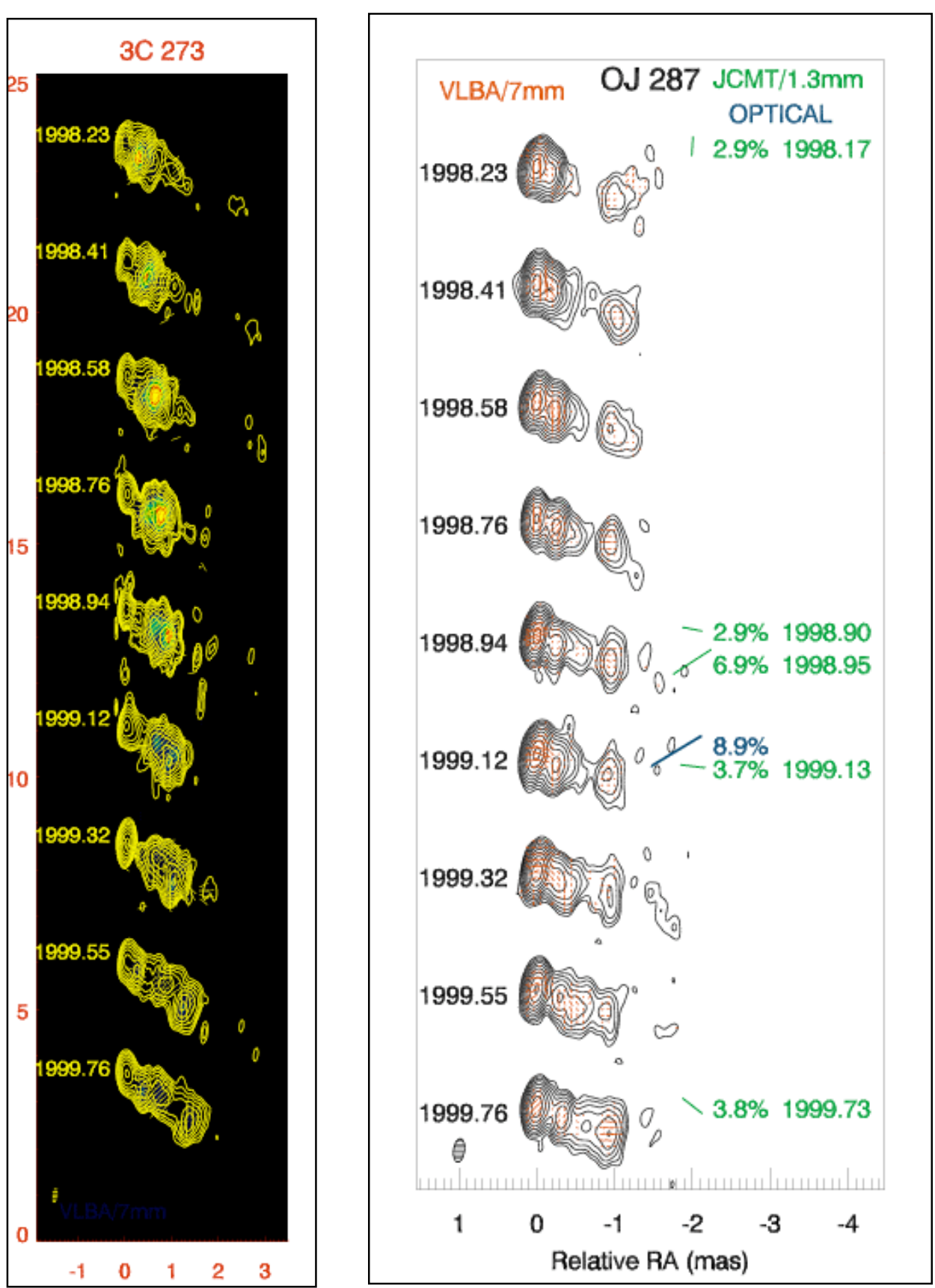

Figure 2. Sequences of VLBA images of the quasar 3C 273 (left) and BL Lac object OJ287 (right) at frequency $43 \mathrm{GHz}$. 


\section{Apparent Speeds}

We have determined the apparent speeds, $\beta_{\text {app }}$, for 109 knots during the three years of the project. Superluminal apparent speeds occur in $82 \%$ of the knots; data for $22 \%$ of these knots reveal deviation from ballistic motion. Figure 3 shows the distributions of apparent speeds in the quasars (53 knots), BL Lac objects (22 knots), and radio galaxies (23 knots).
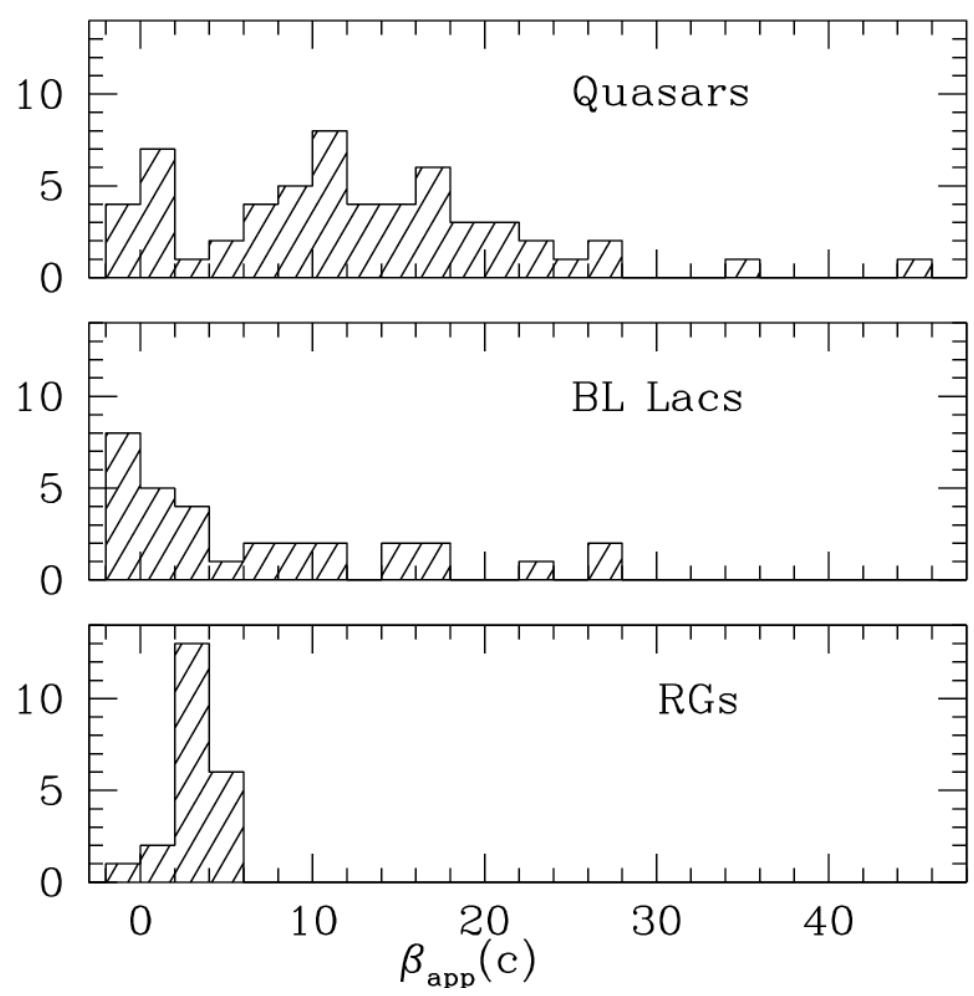

Figure 3. Distributions of the apparent speeds in the quasars, BL Lac objects and radio galaxies.

Figure 3 demonstrates that, in the quasars and BL Lac objects, jet velocities cover a wide range of apparent speeds from subluminal negative values (meaning that the brightness centroids of some components appear to move upstream; such behavior was noted in the $2 \mathrm{~cm}-$ survey [2]) to $\beta_{\text {app }}>30 c \quad\left(\mathrm{H}_{\mathrm{o}}=70 \mathrm{~km} \mathrm{~s}^{-1} \mathrm{Mpc}^{-1}, \Omega_{\mathrm{m}}=0.3, \Omega_{\lambda}=0.7\right)$. The distribution for the quasars has a more prominent high apparent speed tail than the distribution for BL Lac objects, with the highest jet velocity, $\beta_{\text {app }}=(46 \pm 4) c$, measured in the quasar 1510-089. The apparent speeds in the radio galaxies do not exceed $6 c$.

\section{Jet Parameters}

Measurement of apparent speeds is one of the main methods to estimate the actual jet velocity, $\beta$, and to determine the bulk Lorentz factor, $\Gamma=\left(1-\beta^{2}\right)^{-1 / 2}$, and Doppler factor, $\delta=\Gamma^{-1}[1$ $\left.\beta \cos \Theta_{0}\right]^{-1}$, of the jet. The traditional method approximates that the apparent speed has the maximum possible value, corresponding to viewing angle of the jet $\Theta_{0} \sim \Gamma^{-1}$. This gives an 
upper limit to the Lorentz factor, since $\beta_{\text {app }} \leq \Gamma$, and an estimate for the Doppler factor, $\delta \approx \beta_{\text {app }}$ for the minimum possible Lorentz factor.

We have suggested a new method for determining Lorentz and Doppler factors as well as an viewing angle of the jet. The method is based on measurement of the apparent speed of a superluminal knot and its light curve. The latter should be densely sampled for calculating the variability time scale as $\Delta t_{\mathrm{var}}=\mathrm{dt} / \ln \left(\mathrm{S}_{\max } / \mathrm{S}_{\min }\right)$ [9], where $\mathrm{S}_{\max }$ and $\mathrm{S}_{\min }$ are the measured maximum and minimum flux densities, respectively, and $d t$ is the time in years between $\mathrm{S}_{\max }$ and $\mathrm{S}_{\min }$. Figure 4 illustrates the measurement of $\Delta \mathrm{t}_{\mathrm{var}}$ for superluminal knots C14 and C16 in the quasar 3C 279 .
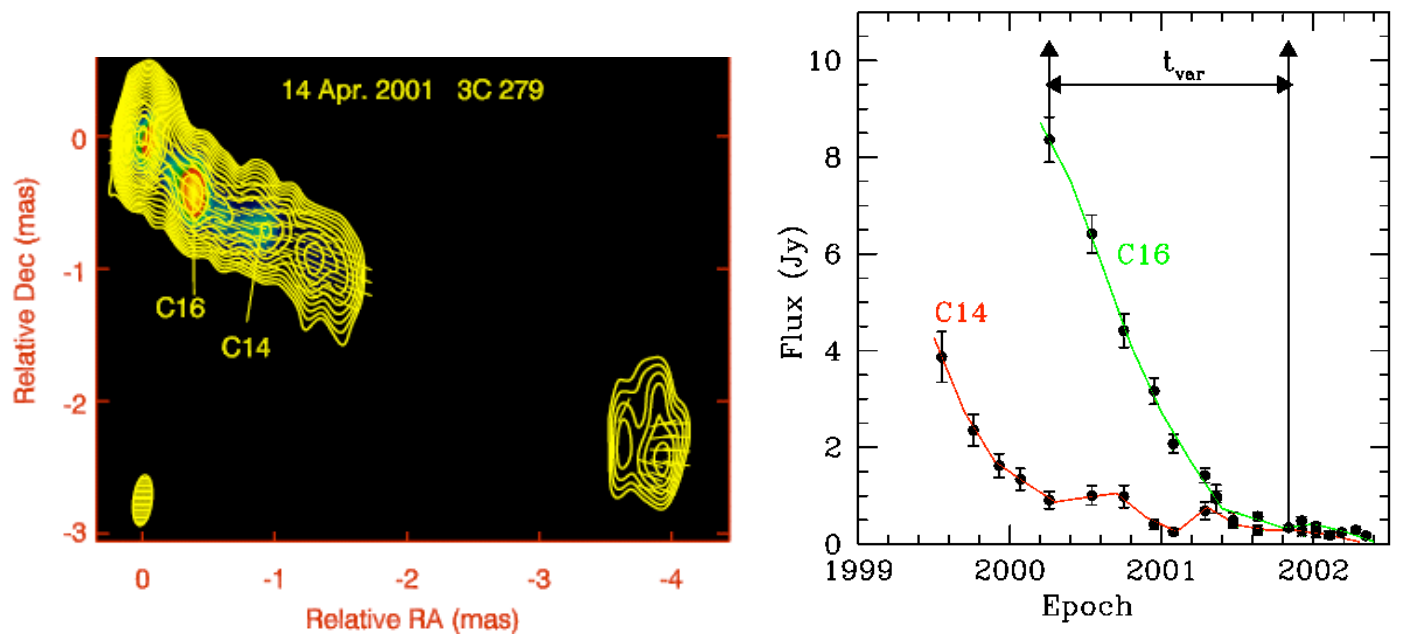

Figure 4. Left: The total and polarized intensity image of the quasar $3 \mathrm{C} 279$ at $43 \mathrm{GHz}$ with components C14 and C16 indicated. Right: The light curves of components C14 and C16.

The variability timescales define the variability Doppler factor as $\delta_{\mathrm{var}}=s D /\left[c \Delta \mathrm{t}_{\mathrm{var}}(1+\mathrm{z})\right]$, where $D$ is the luminosity distance, $s$ is the angular size of the component, equal to $1.6 a$ for a Gaussian with $F W H M=a$ measured at the epoch of maximum flux if the true geometry is similar to a uniform face-on disk. In the case of a point-like knot, we adopt $a=0.1$ mas, which yields an upper limit to $\delta_{\text {var }}$. This definition of the Doppler factor assumes that the variability timescale corresponds to the light-travel time across the knot. This will be true if the radiative cooling time is shorter than the light crossing time, which in turn is shorter than the timescale for cooling by adiabatic expansion. We have calculated the timescales of the variability in size for each non-point-like component and compared these timescales with the relation between flux density and size of a shocked region derived in [10] during the "adiabatic" stage. The comparison confirms that at $43 \mathrm{GHz}$ the decay in flux is driven by radiative losses.

We have calculated the Lorentz factor and viewing angle for each superluminal component, deriving $\beta$ and $\Theta_{0}$ as solutions of the system that combines equations for the apparent speed and variability Doppler factor. While we note that different components in a source show some scatter in Lorentz factor and viewing angle (suggesting changes in actual 
speed and/or jet direction), most of the apparent motions and variability time scales of knots in a given source are roughly consistent with the mean Lorentz factors and viewing angles $\langle\Gamma\rangle$ and $\left\langle\Theta_{0}\right\rangle$, averaged over all the knots in that object for which the required measurements were possible. Figure 5 (left) shows the distributions of Lorentz factors obtained for all superluminal components in the quasars, BL Lac objects, and radio galaxies. The Lorentz factors of the jet flows in the quasars and BL Lac objects range from $\Gamma \sim 5$ to $\Gamma \sim 40$, while the radio galaxies have lower Lorentz factors than the blazars. Figure 5 (right) gives a comparison between the average Lorentz factor for each jet obtained using our method and the maximum apparent speed in the jet that defines the Lorentz factor in the case of the traditional method. Although there is good agreement between the values, it seems that the traditional method overestimates slightly the Lorentz factors for the highest apparent speeds, $>20 \mathrm{c}$.

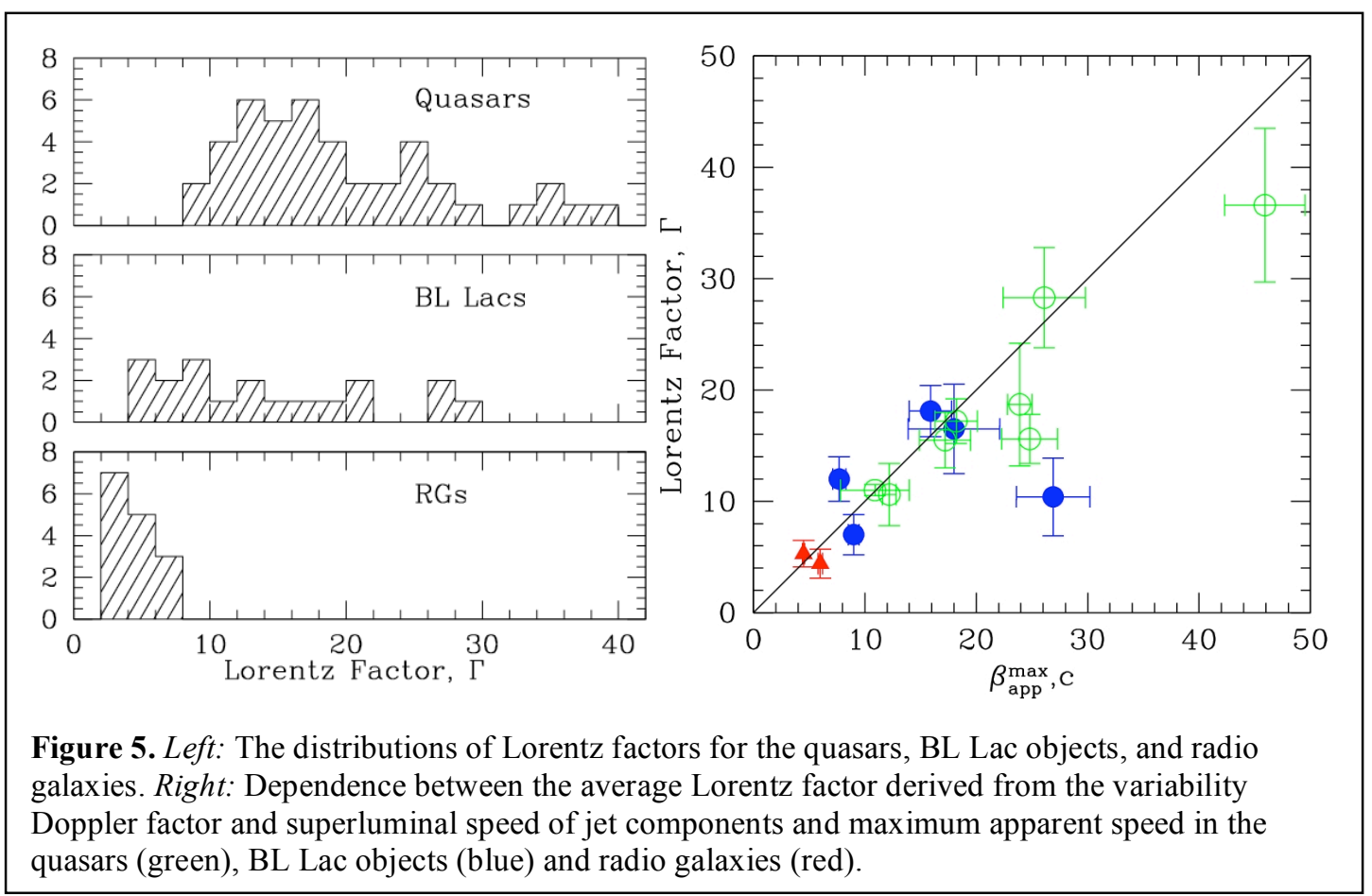

We estimate the projected half opening angle, $\theta_{\mathrm{p}}$, for each source using the ratio between apparent transverse size, $s_{\mathrm{t}}$, of the jet and apparent longitudinal distance, $s_{\mathrm{l}}$, of components: $\theta_{\mathrm{p}}=$ $\tan ^{-1}(\psi)$, where $\psi$ is the slope of the best linear fit to the relation between $s_{\mathrm{t}}$ and $s_{1}$ as defined at the position of each component that is brighter than $1 \%$ of the peak intensity. The values of $s_{\mathrm{t}}$ and $s_{1}$ are estimated as follows: $s_{1}=R$, where $R$ is the observed separation of the component from the core and $s_{\mathrm{t}}=R \sin \left(\left|\Theta_{\text {jet }}-\Theta\right|\right)+a / 2$, where $\Theta_{\text {jet }}$ is the projected direction of the jet as defined by the mean position angle of all sufficiently bright components over all epochs. An example of finding the slope is shown for the quasar 3C 279 in Figure 6 (left). Knowledge of the projected opening angle and viewing angle allow us to estimate the opening angle for each jet: $\theta=2 \theta_{\mathrm{p}}$ $\sin \Theta_{0}$. Figure 6 (right) shows the dependence between the opening angle and inverse Lorentz factor for the jets in our sample, which corresponds to a very good correlation (coefficient of correlation $r=0.83$ ). The best fit of the relation is $\theta=\rho / \Gamma$, where $\rho=0.6 \pm 0.1$. This is consistent 
with theoretical expectations that the opening angle of the jet should be inversely proportional to the Lorentz factor (e.g., [11]).

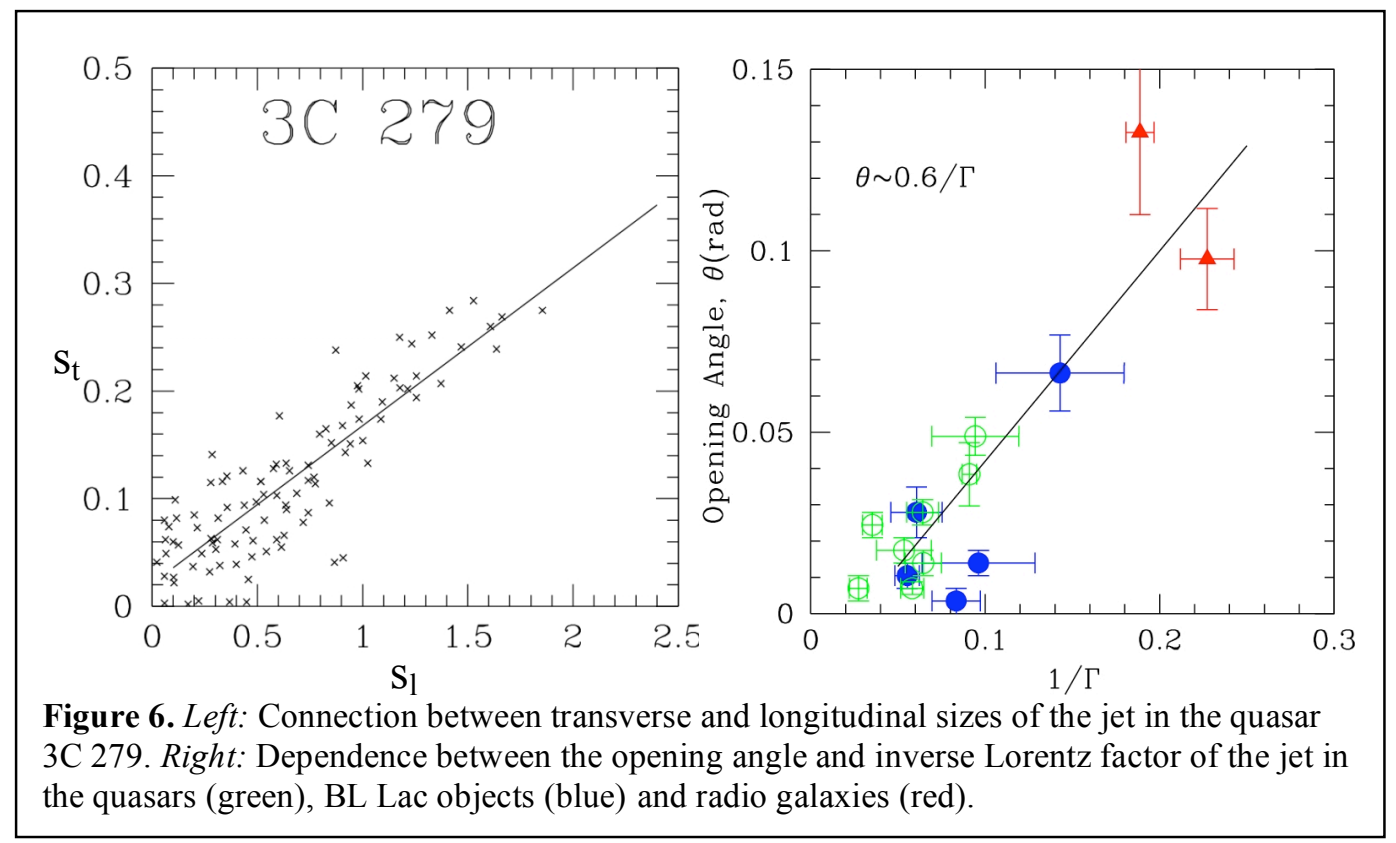

The distributions of Lorentz factors (Fig. 5) suggest higher values of $\Gamma$ in blazars than in radio galaxies and a tentative difference between Lorentz factors of the quasars and BL Lac objects. Figure 6 indicates a connection between the opening angle and inverse Lorentz factor. In addition, Monte-Carlo simulations for flux-limited samples of radio sources predict a connection - although not direct correlation - between the Lorentz factor and viewing angle of the jets: the higher the Lorentz factor, the smaller the viewing angle [12].

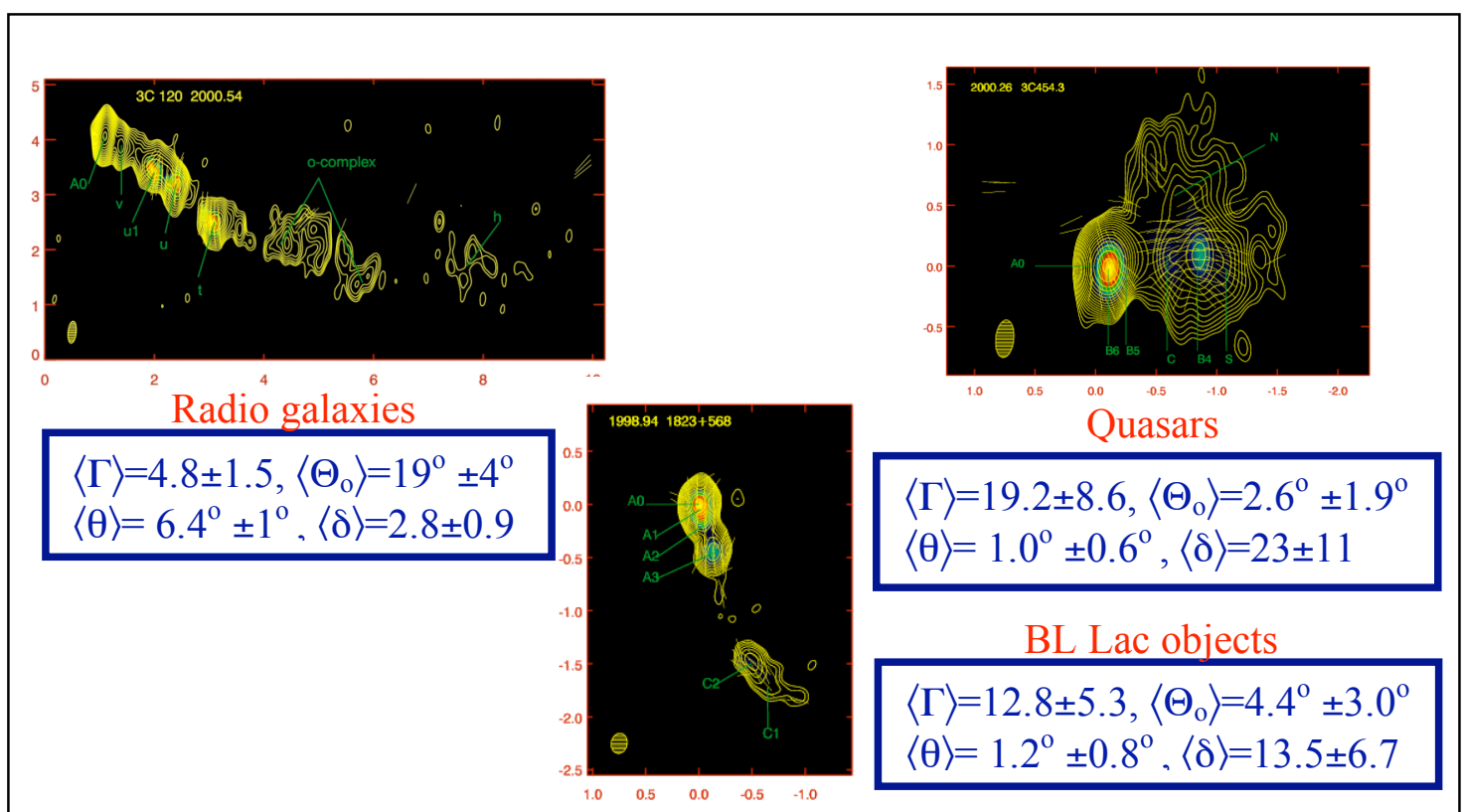

Figure 7. The average values of jet parameters for the radio galaxies, quasars, and BL Lac objects. 
This implies that differences in Lorentz factors for different types of AGNs should lead to differences in other physical parameters of jets. Figure 7 shows a diagram with the values of Lorentz and Doppler factors, viewing and opening angles averaged over the 8 quasars, 5 BL Lac objects, and 2 radio galaxies. Although each subclass has a scatter around the average values and statistically the difference in the parameters of the quasars and BL Lac objects is negligible, the parameter space forms a continuous sequence, with quasars having the highest Lorentz and Doppler factors and smallest viewing and opening angles, while radio galaxies possess the lowest $\Gamma$ and $\delta$ and the largest $\Theta_{0}$ and $\theta$. The BL Lac objects have slightly lower Lorentz and Doppler factors and larger viewing angles than do the quasars.

\section{Polarization Variability}

We have used multifrequency polarization observations to study correlations between total flux, fractional polarization, and polarization position angle at the different wavelengths. Analysis of the polarization variability in the VLBI core at $7 \mathrm{~mm}$ suggests separation of the sample into three groups with low, intermediate, and high variability of polarization in the core: LVP, IVP, and HVP, respectively. The groups are partially associated with the common classification of active galactic nuclei as radio galaxies and quasars with low optical polarization (LVP), BL Lac objects (IVP), and highly optically polarized quasars (HVP). The separation is based on both the variability of the degree and position angle of polarization defined by the variability indices, $V^{\mathrm{p}}{ }_{7 \mathrm{~mm}}$ and $V^{\mathrm{a}}{ }_{7 \mathrm{~mm}}$, respectively. Figure 8 contains the formula for deriving polarization variability indices and a plot of $V^{\mathrm{p}}{ }_{7 \mathrm{~mm}}$ vs. $V^{\mathrm{a}}{ }_{7 \mathrm{~mm}}$ that demonstrates a good correlation between the indices $(r=0.83)$ and boundary values of $V^{\mathrm{p}}{ }_{7 \mathrm{~mm}}$ and $V^{\mathrm{a}}{ }_{7 \mathrm{~mm}}$ used for separation into groups. According to the polarization-based classification, the LVP group includes two radio galaxies and the quasar 3C 273, the IVP group consists of four (out of five)

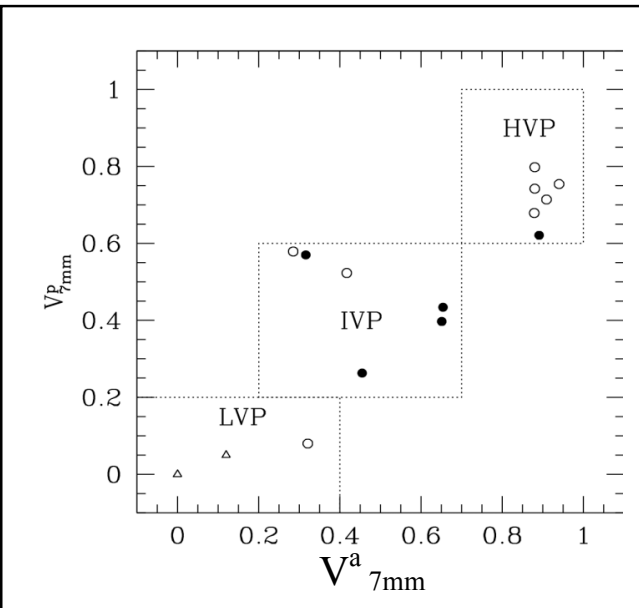

Figure 8. Connection between polarization and position angle variability indices in the VLBI core at $7 \mathrm{~mm}$. Symbols denote the quasars (open circles), BL Lac objects (filled circles), and radio galaxies (triangles).

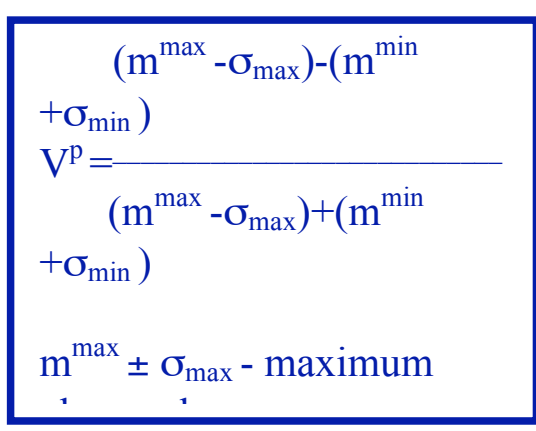

$\mathrm{V}^{\mathrm{a}}=\frac{|\Delta \chi|-\left(\sigma_{1}^{2}+\sigma_{2}^{2}\right)^{1 / 2}}{90}$
$|\Delta \chi|-$ observed range of
EVPAs
$\sigma_{1}, \sigma_{2}-$ the uncertainties in


BL Lac objects and two highly optically polarized quasars, 3C 279 and 3C 345 . The majority of the quasars (five out of eight) and one BL Lac object, OJ287, form the HVP group.

We have compared the degree of polarization in the core at $43 \mathrm{GHz}$ with the degree of polarization from the whole source at higher frequencies using the closest observations (within 1-2 days) at different wavelengths. Figure 9 (left) shows the results of the comparison. There is a statistically significant correlation between fractional polarization in the VLBI core and degree of polarization at shorter wavelengths. The optical polarization maintains the strongest connection to the polarization in the VLBI core $(r=0.87)$. This result confirms the strong correlation between the polarization level of the radio core at $7 \mathrm{~mm}$ and overall optical polarization found by Lister \& Smith [16] in a sample of quasars with both high and low

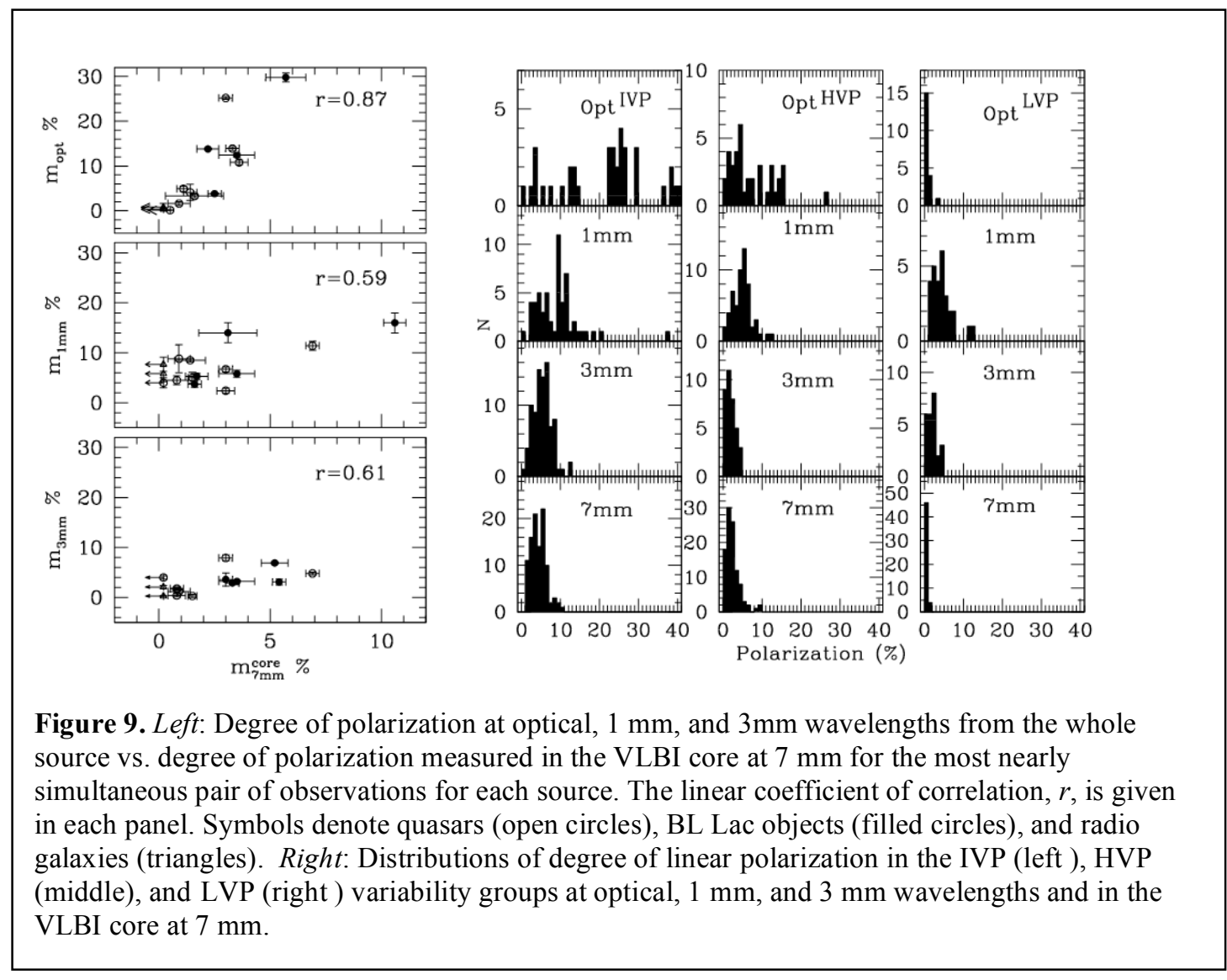

optical polarization.

Figure 9 (right) presents the distributions of fractional polarization for all of our measurements, separated according to the variability groups. Comparison of the distributions shows that (1) the peak of the distribution in the IVP group occurs at a higher percentage polarization than for the other two groups, independent of wavelength, and (2) for a given variability group, the peak of the distribution shifts to higher fractional polarization at shorter wavelengths except for the LVP sources, where the optical polarization is similar to the polarization in the VLBI core.

According to our analysis of jet kinematics, the quasars, BL Lac objects, and radio galaxies possess different Lorentz factors. We have compared polarization variability indices 
with $\Gamma$ of the jet for each source. Figure 10 shows the dependence between bulk Lorentz factor and polarization variability index. There is a correlation between $\Gamma$ and $V^{\mathrm{p}}{ }_{\text {opt }}(r=0.53)$ and between $\Gamma$ and $V^{p}{ }_{7 \mathrm{~mm}}(r=0.72)$, while $V^{\mathrm{p}}{ }_{1 \mathrm{~mm}}$ does not show any correlation $(r=-0.12)$. In the plane-wave shock model, the correlation is expected if the minimum degree of polarization corresponds to unshocked plasma and the maximum to faster, shocked plasma.

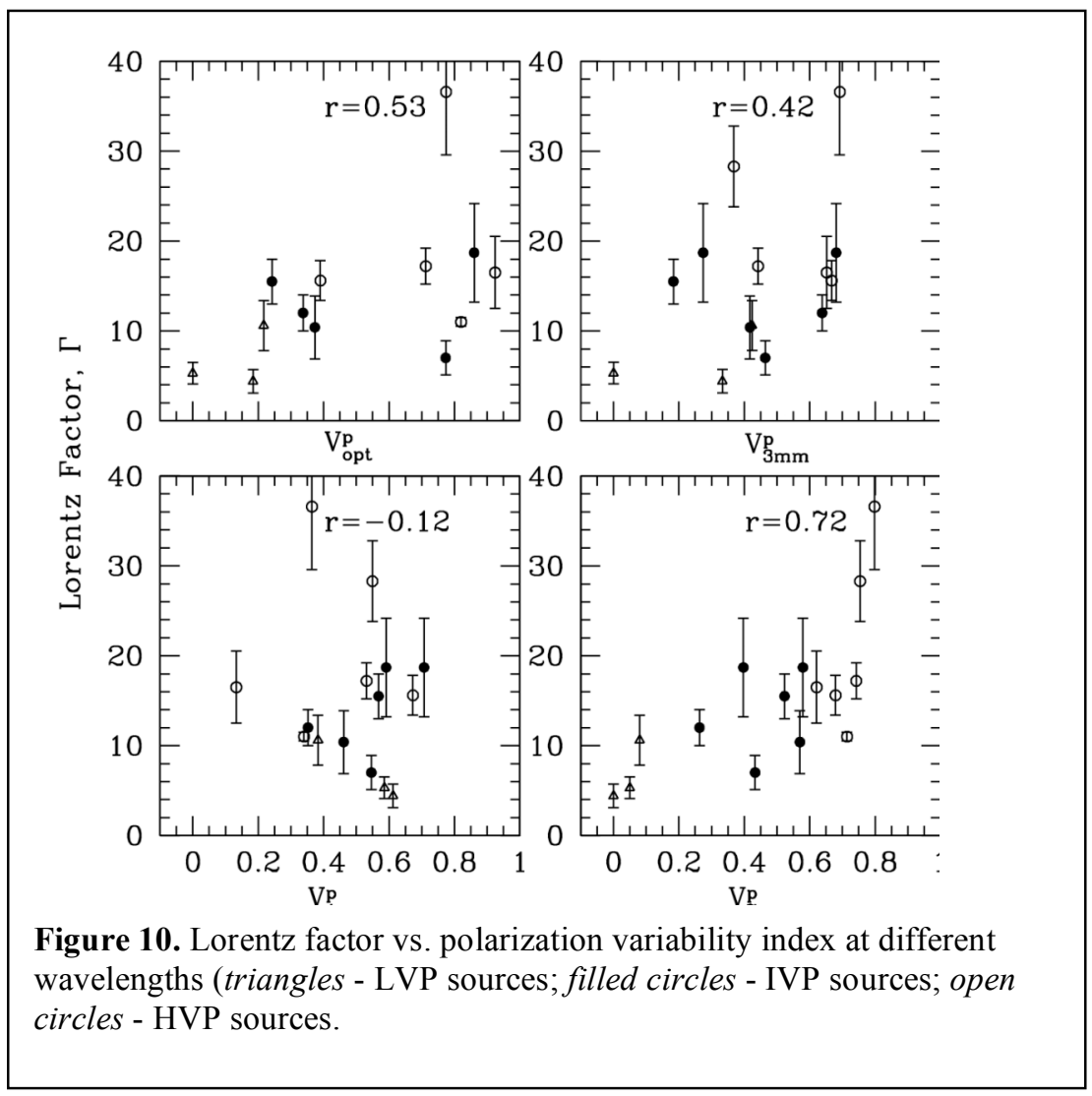

\section{Faraday Rotation in the Inner Jet}

Analysis of position angles of polarization at various millimeter wavelengths (grouped according to closest dates) reveals that the direction of millimeter-wave polarization rotates with wavelength, $\lambda$. In the HVP and IVP sources we used the EVPA at $7 \mathrm{~mm}$ corresponding to that of the VLBI core only, while in the LVP sources the Stokes parameters $I, Q$, and $U$ are

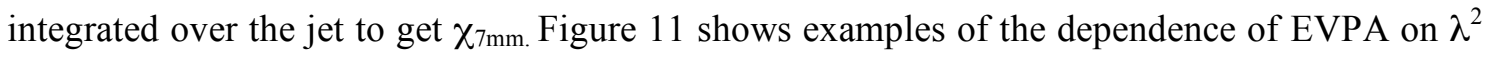
in the HVP and IVP sources, supporting the hypothesis that the rotation can be attributed to Faraday rotation by a foreground screen close to the VLBI core $[13,14]$. We define the rotation measure $(R M)$ by assuming that the direction of polarization at $1 \mathrm{~mm}$ is unrotated and that the emission from 1 to $7 \mathrm{~mm}$ propagates through the same screen. This $R M$ should correspond to the rotation measure in the VLBI core after taking in account that we have selected for $R M$ measurements epochs at which the jets of the HVP and IVP blazars have not possessed highly polarized components at $7 \mathrm{~mm}$ beyond the core, which provides some insurance that the polarization determined at 1 and $3 \mathrm{~mm}$ from the whole source comes mostly from the core. We 
have determined the intrinsic rotation measure $R M_{o}=R M(1+z)^{2}$ for each source in the HVP and IVP groups and averaged the values over each group. As a result, the IVP sources have lower $R M s,\left\langle R M_{o}\right\rangle=(1.1 \pm 0.3) \times 10^{4} \mathrm{rad} \mathrm{m}^{-2}$, than the HVP sources, $\left\langle R M_{o}\right\rangle=(8.5 \pm 6.5) \times 10^{4} \mathrm{rad} \mathrm{m}^{-2}$.
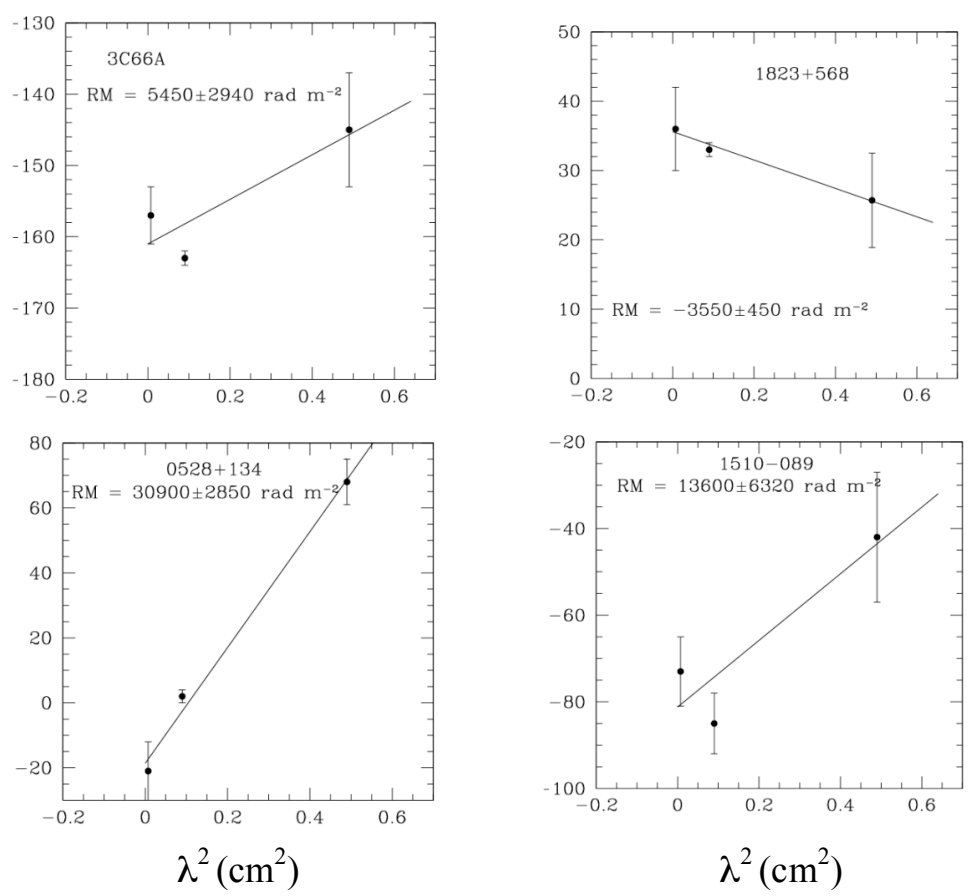

Figure 11. Dependence of polarization position angle on square of wavelength in the IVP and HVP sources. The solid line represents an approximation of the dependence by a $\lambda^{2}$ Faraday rotation law.

Comparison of the degree of polarization integrated over the jet at $7 \mathrm{~mm}$ with the degree of polarization from the whole source at $1 \mathrm{~mm}$ in the LVP sources (Fig. 12) shows that the values at the two wavelengths are very similar. As noted previously, the VLBI core in the LVP sources is unpolarized (the polarized intensity is lower than the noise level, $\sim 3-5 \mathrm{mJy} / \mathrm{beam}$ ). In addition, the high $R M$ values are obtained close to the core in 3C 273 [14]. These properties can be interpreted as in the LVP sources: the 1 and $3 \mathrm{~mm}$ cores have low polarization as well. The low polarization of the core could be the result of either fine-scale turbulence or depolarization by a very thick, inhomogeneous foreground screen with $R M>5 \times 10^{5} \mathrm{rad} \mathrm{m}^{-2}$, as suggested by Attridge et al. [14]. The latter implies that the thickness of the foreground screen near the mmwave core is highest in the LVP sources and decreases in the HVP and IVP blazars, with the minimum $R M$ in the IVP sources. This may be a reason why BL Lac objects, which form the majority of the IVP group, do not contain prominent emission lines. 


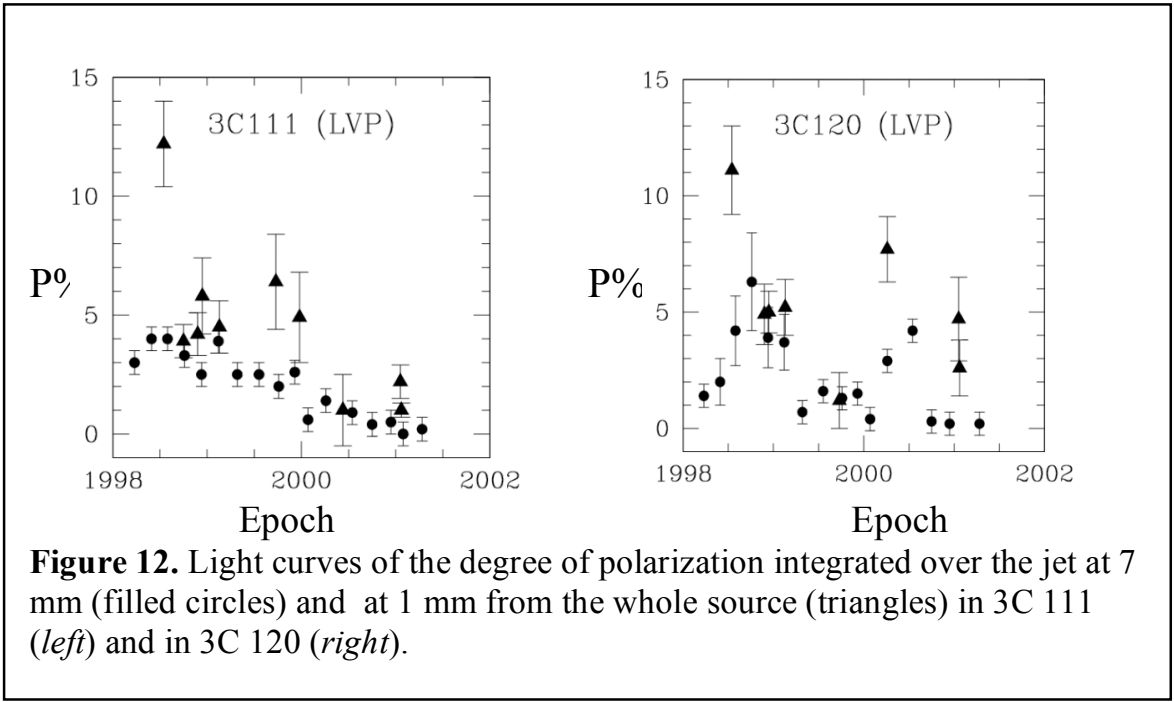

\section{Comparison between the Jet and Polarization Directions}

We have compared the polarization position angle, $\chi_{\lambda}$, for each polarization measurement with the position angle of the jet projected on the sky, $\Theta_{\text {jet }}$, corresponding to the nearest VLBA epoch to a given polarization measurement. Figure 13 gives the distributions of the offsets between direction of polarization and jet axis, $\left|\chi_{\lambda}-\Theta_{\text {jet }}\right|$, for polarization measured at the different wavelengths, $\lambda$, and for the different groups of object. In the case of the IVP and HVP sources, $\chi_{7 \mathrm{~mm}}$ corresponds to the core and is corrected for $R M$, while in the LVP group $\chi_{7 \mathrm{~mm}}$ is integrated over the jet.

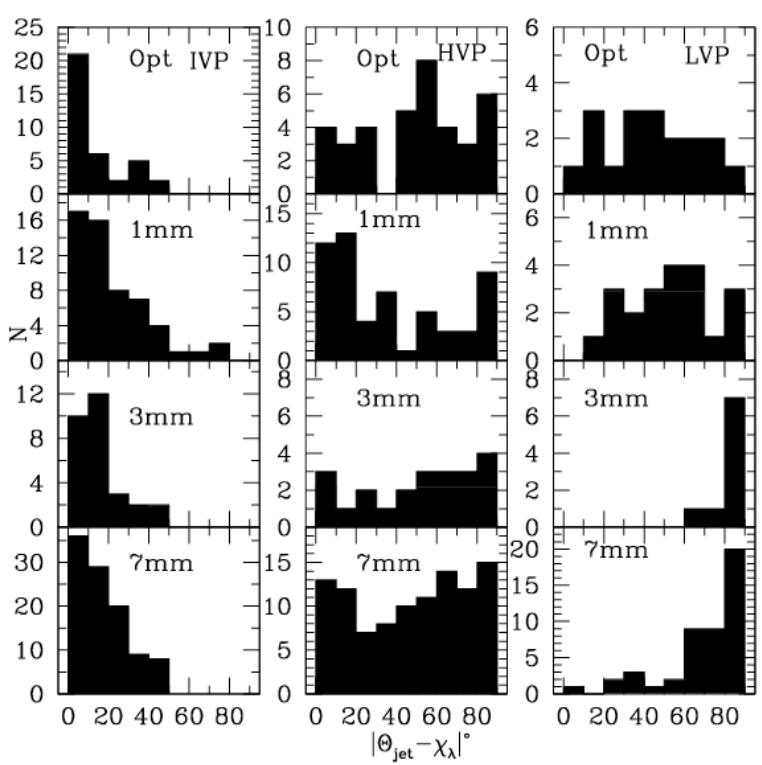

Figure 13. Distribution of offsets between polarization position angle and jet direction. 
Figure 13 shows obvious alignment of the electric vector with the jet direction in the IVP sources. In contrast, the HVP sources do not show any significant relation between EVPA and jet direction. The difference between the distributions of the IVP and HVP sources is similar to the difference between the distributions of core electric vector offsets from the jet direction found by Lister \& Homan [15] for BL Lac objects and quasars, respectively, in the Monitoring of Jets in AGNs with VLBA Experiments survey at $2 \mathrm{~cm}$. In the LVP sources the distributions of $\left|\chi_{\lambda}-\Theta_{\text {jet }}\right|$ at optical and $1 \mathrm{~mm}$ wavelengths do not support a connection of the EVPA with the jet direction, while the polarization position angle at $3 \mathrm{~mm}$ and in the inner jet at $7 \mathrm{~mm}$ clearly reveals a preferential direction perpendicular to the jet.

The properties of polarization position angle with respect to the jet direction are distinct for each group, independent of wavelength and similar at different wavelengths for a given group. These differences can be caused by differences in jet physical parameters: 1) intrinsic difference in the structure of the magnetic field (large scale in the IVP, fine structure in the HVP, the finest structure in the LVP group); 2) difference in the amount of hot gas in the core region; 3 ) difference in morphology of the inner jet (straight symmetric jets in the IVP; curved asymmetric jets in the HVP; wide, with a velocity gradient across the jet, jets in the LVP group); 4) difference in shock strengths as suggested by Lister \& Smith [16].

\section{Where does polarized emission at different wavelengths originate?}

Analysis of polarization properties at optical and mm-wavelengths along with high resolution total and polarized intensity imaging shows that 1) for simultaneous observations at different wavelengths the degree of polarization in the VLBI core at $7 \mathrm{~mm}$ strongly correlates with the degree of optical polarization from the whole source; 2) jets with the highest bulk Lorentz factors have the largest optical and $7 \mathrm{~mm}$ (in the core) polarization variability indices, while $\mathrm{V}^{\mathrm{p}}{ }_{1 \mathrm{~mm}}$ does not correlate with $\Gamma$; and 3) in the HVP and IVP blazars there is the best alignment between EVPAs at optical wavelengths and in the VLBI core for simultaneous observations. A good correlation between polarization properties at optical wavelengths and in the $7 \mathrm{~mm}$ core suggests that the polarized emission at both wavelengths is caused by the same mechanism, most likely shocks. The partial discrepancy between polarization properties at 1 $\mathrm{mm}$ suggests that another emission component in addition to transverse shocks is prominent at 1 $\mathrm{mm}$. A primary candidate is the "true" core, i.e., the bright, narrow end of the jet when observed at a wavelength where the emission is completely optically thin (Fig. 14). At wavelengths of 3 $\mathrm{mm}$ and longer, what appears to be the core is most likely a location at or outside the point where the optical depth $\tau(v) \sim 1$. The fact that the optical polarization does show the correlation, as well as the best alignment of position angle with the electric vector in the core at $7 \mathrm{~mm}$, implies that most of the nonthermal optical emission arises in shocks close to the $7 \mathrm{~mm}$ core rather than in the "true" core. This suggests that the "true" core does not possess relativistic electrons energetic enough to produce the optical synchrotron emission.

\section{Acknowledgments}

The original work reported here was supported in part by National Science Foundation grant AST-0406865. This work was supported by NASA APP grant NNX08AJ64G. The VLBA 
and VLA are instruments of the National Science Foundation operated by the National Radio Astronomy Observatory under cooperative agreement by Associated Universities Inc.

\section{References}

[1] S.G. Jorstad et al., The Multiepoch Very Long Baseline Array Observations of EGRET-detected Quasars and BL Lacertae Objects: Superluminal Motion of Gamma-Ray Bright Blazars, ApJS 134, 181-240 (2001).

[2] K.I. Kellermann et al., Sub-Milliarcsecond Imaging of Quasars and Active Galactic Nuclei. III. Kinematics of Parsec-scale Radio Jets, ApJ 609, 539-563 (2004).

[3] B.G. Piner et al., The Fastest Relativistic Jets: VLBA Observations of Blazars with Apparent Speed Exceeding 25c, ApJ 640, 196-203 (2006).

[4] D.L. Meier, S. Koide, \& Y. Uchida, Magnetohydrodynamic Production of Relativistic Jets, Science 291, 84-92 (2001).

[5] J.C. McKinney, General Relativistic Magnetohydrodynamic Simulations of the Jet Formation and Large-Scale Propagation from Black Hole Accretion Systems, MNRAS 368, 1561-1582 (2006).

[6] M. Sikora et al., Are Quasars Jets Dominated by Poynting Flux?, ApJ 625, $72-77$ (2005).

[7] S.G. Jorstad et al., Polarimetric Observations of 15 Active Galactic Nuclei at High Frequencies: Jet Kinematics from Bimonthly Monitoring with the Very Long Baseline Array, AJ 130, 1418-1465 (2005).

[8] S.G. Jorstad et al., Polarimetric Observations of 15 Active Galactic Nuclei at High Frequencies: Correlated Polarization Behavior at Different wavelengths, AJ 134, 799-824 (2007).

[9] G.R. Burbidge, T.W. Jones, \& S.L. O’Dell, Physics of Compact Nonthermal Sources. III Energetic Considerations, ApJ 193, 43-54 (1974).

[10] A.P. Marscher \& W.K. Gear, Models for High-Frequency Radio Outbursts in Extragalactic Sources, with Application to the Early 1983 Millimeter-to-Infrared Flare of 3 C 273, ApJ 298, 114127 (1985).

[11] R.D. Blandford \& A. Königl, Relativistic Jets as Compact Radio Sources, ApJ 232, 34-48 (1979).

[12] M.L. Lister \& A.P. Marscher, Statistical Effects of Doppler Beaming and Malmquist Bias on FluxLimited Samples of Compact Radio Sources, ApJ 476, 572-588 (1997).

[13] R.T. Zavala \& G.B. Taylor, A View through Faraday's Fog. II. Parsec-Scale Measures in 40 Active Galactic Nuclei, ApJ 612, 749-779 (2004).

[14] J.M. Attridge, J.F.C. Wardle, \& D.C. Homan, Concurrent 43 and 86 GHz Very Long Baseline Polarimetry of 3C 273, ApJ 633, L85-L88 (2005).

[15] M.L. Lister \& D.C. Homan, MOJAVE: Monitoring of Jets in Active Galactic Nuclei with VLBA Experiments. I. First-Epoch 15 GHz Linear Polarization Images, AJ 130, 1389-1417 (2005).

[16] M.L. Lister \& P.S. Smith, Intrinsic Differences in the Inner Jets of High and Low Optically Polarized Radio Quasars, ApJ 541, 66-87 (2000). 